\title{
Classification of the centers, of their cyclicity and isochronicity for two classes of generalized quintic polynomial differential systems
}

\author{
Jaume Llibre and Clàudia Valls
}

\begin{abstract}
In this paper we classify the centers localized at the origin of coordinates, the cyclicity of their Hopf bifurcation and their isochronicity for the polynomial differential systems in $\mathbb{R}^{2}$ of degree $d$ that in complex notation $z=x+i y$ can be written as

$$
\dot{z}=(\lambda+i) z+(z \bar{z})^{\frac{d-5}{2}}\left(A z^{4+j} \bar{z}^{1-j}+B z^{3} \bar{z}^{2}+C z^{2-j} \bar{z}^{3+j}+D \bar{z}^{5}\right),
$$

where $j$ is either 0 or $1, d$ is an arbitrary odd positive integer greater than or equal to five, $\lambda \in \mathbb{R}$, and $A, B, C, D \in \mathbb{C}$. Note that if $d=5$ we obtain special families of quintic polynomial differential systems.
\end{abstract}

Mathematics Subject Classification (2000). 34C05, 58F14.

Keywords. Center, Isochronous center, Cyclicity, Polynomial vector fields.

\section{Introduction and statement of the main results}

One of the main problems in the qualitative theory of real planar polynomial differential systems is the center-focus problem; i.e. to distinguish when a singular point is either a focus or a center. The definition of center goes back to Poincaré in [16]; i.e. a singular point of a vector field on the real plane surrounded by a neighborhood fulfilled of periodic orbits with the unique exception of the singular point. This article deals with the center-focus problem for a class of polynomial differential systems which generalizes two families of quintic polynomial differential systems with homogeneous nonlinearities.

The classification of centers for the polynomial differential systems started with the quadratic ones with the works of Dulac [9], Kapteyn [12,13], Bautin [2], and references therein. Schlomiuk et al. in [17] described a brief history of the problem of the center in general and of the quadratic case in particular. Here we are mainly interested in finding new families of centers of polynomial differential systems of arbitrary degree and in study their cyclicity and isochronicity. 
In our computations we use complex notation for real planar polynomial differential systems for finding such new families of centers, so we will be interested in the expression of the Poincaré-Liapunov constants in complex notation. The reason for using the complex notation is that it simplifies the computations and the expressions of these constants. As far as we know the first in using this complex notation was Żołądek in [19] and [20].

There are many partial results for the centers of polynomial differential systems of degree larger than 2. Here we only mention the ones which are more close to our results. For example, the centers for cubic polynomial differential systems of the form linear with homogeneous nonlinearities of degree 3 were characterized by Malkin [14] and Vulpe and Sibirskii [18]. When the homogeneous nonlinearities are of degree $k>3$ the centers are not classified, but there are partial results for $k=4,5$ see for instance Chavarriga and Giné [3,4], respectively.

In this paper we consider the polynomial differential systems in the real $(x, y)$-plane that has a singular point at the origin with eigenvalues $\lambda \pm i$ and that can be written as

$$
\dot{z}=(\lambda+i) z+(z \bar{z})^{\frac{d-5}{2}}\left(A z^{4+j} \bar{z}^{1-j}+B z^{3} \bar{z}^{2}+C z^{2-j} \bar{z}^{3+j}+D \bar{z}^{5}\right),
$$

where $j$ is either 0 , or $1, z=x+i y, d$ is an arbitrary odd positive integer greater than or equal to five, $\lambda \in \mathbb{R}$, and $A, B, C, D \in \mathbb{C}$. When $j=0$ we are considering the class of systems

$$
\dot{z}=(\lambda+i) z+(z \bar{z})^{\frac{d-5}{2}}\left(A z^{4} \bar{z}+B z^{3} \bar{z}^{2}+C z^{2} \bar{z}^{3}+D \bar{z}^{5}\right),
$$

and when $j=1$ we are considering the class of systems

$$
\dot{z}=(\lambda+i) z+(z \bar{z})^{\frac{d-5}{2}}\left(A z^{5}+B z^{3} \bar{z}^{2}+C z \bar{z}^{4}+D \bar{z}^{5}\right) .
$$

The vector field associated to system (1) is formed by the linear part $(\lambda+i) z$ and by a homogeneous polynomial of degree $d$ formed by four monomials in complex notation. Since the eigenvalues at the singular point located at the origin of system (1) are $\lambda \pm i$, the origin is either a weak focus or a center if $\lambda=0$, see for instance $[1,15]$.

For systems (1) we want to determine the conditions that ensure that the origin is a center or an isochronous center. Of course these systems for $d=5$ coincides with two families of quintic polynomial differential systems of the form linear terms with quintic homogeneous nonlinearities. So we call these classes of polynomial differential systems (1) of degree $d \geq 5$ the generalized quintic systems.

The first main result in this paper is the following theorem.

Theorem 1. For $d \geq 5$ odd the following statements holds for system (1).

(a) It has a center at the origin if and only if one of the following three conditions hold.

(a.1) $\lambda=b_{1}=(4+j) A /(2-j)+\bar{C}=0$,

(a.2) $\lambda=b_{1}=\operatorname{Im}(A C)=\operatorname{Im}\left(A^{3} D\right)=\operatorname{Im}\left(\bar{C}^{3} D\right)=0$ when $j=0$.

(a.3) $\lambda=b_{1}=\operatorname{Im}(A C)=\operatorname{Re}\left(A^{3} D^{2}\right)=\operatorname{Re}\left(\bar{C}^{3} D^{2}\right)=0$ when $j=1$. 
Moreover the centers satisfying conditions (a.1) are Hamiltonian, and the ones satisfying conditions (a.2) and (a.3) are reversible.

(b) It has an isochronous center at the origin if and only if one of the following two conditions hold.

(b.1) $\lambda=B=D=0, C=\bar{A}$,

(b.2) $\quad \lambda=B=D=0, C=(3+2 j-d) \bar{A} /(1+2 j+d)$ when $j=0,1$,

(b.3) $\lambda=B=C=0, D=-\bar{A}^{2} / A, d=7$ and $j=0$.

We remark that the families of centers described in Theorem 1 are new, and that the families of isochronous centers are also new because they did not appear in the good survey of Chavarriga and Sabatini [5] on isochronous centers.

Now we want to study the maximum number of limit cycles bifurcating from the origin for the class of polynomial differential systems (1). If we denote by $E_{d}$ the class of all systems of degree $d$ of the form (1) we say that the origin of any systems $\dot{z}=w(z, \bar{z})$ with $w \in E_{d}$ has cyclicity $k$ with respect to $E_{d}$ if any perturbation of this system inside the class $E_{d}$ has at most $k$ limit cycles in a small neighborhood of the origin, and $k$ is reached for some perturbation.

Theorem 2. The cyclicity of the equilibrium point $z=0$ of system (1) when $j=0$ with respect to $E_{d}$ is less than or equal to five for $d \geq 29$ odd, and less than or equal to six for $d \in\{5,7, \ldots, 27\}$. More precisely the cyclicity is

(a) 0 for $\lambda \neq 0$;

(b) 1 for $\lambda=0, b_{1} \neq 0$;

(c) 2 for $\lambda=b_{1}=0 \neq 0, \operatorname{Im}(A C) \neq 0$;

(d) 3 for

(d.1) $\lambda=b_{1}=\operatorname{Im}(A C)=0,(d-7) A+(d+5) \bar{C} \neq 0$;

(d.2) $\quad \lambda=b_{1}=\operatorname{Im}(A C)=0,(d-3) A+(d+1) \bar{C} \neq 0 ;$

(e) 4 for

(e.1) $\lambda=b_{1}=\operatorname{Im}(A C)=(d-7) A+(d+5) \bar{C}=0, b_{2} \neq 0$;

(e.2) $\lambda=b_{1}=\operatorname{Im}(A C)=(d-3) A+(d+1) \bar{C}=0, b_{2} \neq 0$;

(f) 5 for

(f.1) $\lambda=B=\operatorname{Im}(A C)=(d-7) A+(d+5) \bar{C}=0, A^{2} u+\bar{C}^{2} v \neq 0$;

(f.2) $\lambda=B=\operatorname{Im}(A C)=(d-3) A+(d+1) \bar{C}=0, A^{2} u+\bar{C}^{2} v \neq 0$;

(g) 6 for $\lambda=B=\operatorname{Im}(A C)=(d-7) A+(d+5) \bar{C}=A^{2} u+\bar{C}^{2} v=0, C \neq 0$, $d \in\{5,7, \ldots, 27\}$.

Theorem 3. The cyclicity of the equilibrium point $z=0$ of system (1) when $j=1$ with respect to $E_{d}$ is less than or equal to five for $d \geq 41$ odd, and less than or equal to six for $d \in\{5,7, \ldots, 39\}$. More precisely the cyclicity is

(a) 0 for $\lambda \neq 0$;

(b) 1 for $\lambda=0, b_{1} \neq 0$;

(c) 2 for $\lambda=b_{1}=0 \neq 0, \operatorname{Im}(A C) \neq 0$;

(d) 3 for

(d.1) $\lambda=b_{1}=\operatorname{Im}(A C)=0,(d-17) A+(d+15) \bar{C} \neq 0 ;$ 
(d.2) $\quad \lambda=b_{1}=\operatorname{Im}(A C)=0,(d-7) A+(d+5) \bar{C} \neq 0 ;$

(e) 4 for

(e.1) $\lambda=b_{1}=\operatorname{Im}(A C)=(d-17) A+(d+15) \bar{C}=0, b_{2} \neq 0$;

(e.2) $\lambda=b_{1}=\operatorname{Im}(A C)=(d-7) A+(d+5) \bar{C}=0, b_{2} \neq 0$;

(f) 5 for

(f.1) $\lambda=B=\operatorname{Im}(A C)=\left((d-17) A+(d+15) \bar{C}=0, A^{2} u+\bar{C}^{2} v \neq 0\right.$;

(f.2) $\lambda=B=\operatorname{Im}(A C)=(d-7) A+(d+5) \bar{C}=0, A^{2} u+\bar{C}^{2} v \neq 0$;

(g) 6 for

(g.1) $d \in\{7, \ldots, 39\}, \lambda=B=\operatorname{Im}(A C)=((d-17) A+(d+15) \bar{C}=$ $A^{2} u+\bar{C}^{2} v=0, D \neq 0$

(g.2) $\quad d=5, \lambda=B=3 A-5 \bar{C}=16|C|^{2}-9|D|^{2}=0, \operatorname{Re}(D) \neq 0$.

We have divided the paper as follows. In Sect. 2 we introduce some preliminaries that will be used through all the paper. In Sect. 3 we provide the proof of Theorem 1(a), while the proof of Theorem 1(b) is given in Sect. 4. Finally the proof of Theorems 2 and 3 is given in Section 5 .

\section{Preliminaries}

The resolution of Theorem 1 implies the effective computation of the Liapunov constants as well as of the period constants. We write

$$
A=a_{1}+i a_{2}, \quad B=b_{1}+i b_{2}, \quad C=c_{1}+i c_{2}, \quad D=d_{1}+i d_{2} .
$$

Indeed writing (1) in polar coordinates, i.e. doing the change of variables $r^{2}=z \bar{z}$ and $\theta=\arctan (\operatorname{Im} z / \operatorname{Re} z)$, system (1) becomes

$$
\frac{d r}{d \theta}=\frac{\lambda r+F(\theta) r^{d}}{1+G(\theta) r^{d-1}},
$$

where

$$
\begin{aligned}
F(\theta)= & \left(a_{1}+c_{1}\right) \cos (2(1+j) \theta)-\left(a_{2}-c_{2}\right) \sin (2(1+j) \theta)+b_{1} \\
& +d_{1} \cos (6 \theta)+d_{2} \sin (6 \theta), \\
G(\theta)= & \left(a_{2}+c_{2}\right) \cos (2(1+j) \theta)+\left(a_{1}-c_{1}\right) \sin (2(1+j) \theta)+b_{2} \\
& +d_{2} \cos (6 \theta)-d_{1} \sin (6 \theta) .
\end{aligned}
$$

Clearly Eq. (2) is well defined in a sufficiently small neighborhood of the origin. Therefore if system (1) has a center at the origin, then Eq. (2) defined in the plane $(r, \theta)$ when $\dot{\theta}>0$ also has a center at the origin.

The transformation $(r, \theta) \mapsto(\rho, \theta)$ defined by

$$
\rho=\frac{r^{d-1}}{1+G(\theta) r^{d-1}}
$$

is a diffeomorphism from the region $\dot{\theta}>0$ into its image. As far as we know the first in use this transformation was Cherkas in [7]. If we write Eq. (2) in the 
variable $\rho$, we obtain the following Abel differential equation

$$
\begin{aligned}
\frac{d \rho}{d \theta}= & (d-1) G(\theta)[\lambda G(\theta)-F(\theta)] \rho^{3} \\
& +\left[(d-1)(F(\theta)-2 \lambda G(\theta))-G^{\prime}(\theta)\right] \rho^{2}+(d-1) \lambda \rho \\
= & A(\theta) \rho^{3}+B(\theta) \rho^{2}+C \rho .
\end{aligned}
$$

These kind of differential equations appeared in the studies of Abel on the theory of elliptic functions. For more details on Abel differential equations, see $[6,10,11]$.

The solution $\rho(\theta, \gamma)$ of (4) satisfying that $\rho(0, \gamma)=\gamma$ can be expanded in a convergent power series of $\gamma \geq 0$ sufficiently small. Thus

$$
\rho(\theta, \gamma)=\rho_{1}(\theta) \gamma+\rho_{2}(\theta) \gamma^{2}+\rho_{3}(\theta) \gamma^{3}+\cdots
$$

with $\rho_{1}(\theta)=1$ and $\rho_{k}(0)=0$ for $k \geq 2$. Let $P:\left[0, \gamma_{0}\right] \rightarrow \mathbb{R}$ be the Poincaré map defined by $P(\gamma)=\rho(2 \pi, \gamma)$ and for a convenient $\gamma_{0}>0$. Then, the values of $\rho_{k}(2 \pi)$ for $k \geq 2$ control the behavior of the Poincaré map in a neighborhood of $\rho=0$. Clearly system (1) has a center at the origin if and only if $\rho_{1}(2 \pi)=1$ and $\rho_{k}(2 \pi)=0$ for every $k \geq 2$. Assuming that $\rho_{2}(2 \pi)=\cdots=\rho_{m-1}(2 \pi)=0$ we say that $v_{m}=\rho_{m}(2 \pi)$ is the $m$-th Liapunov or Liapunov-Abel constant of system (1), or simply the Liapunov constant of system (1).

The set of coefficients for which all the Liapunov constants vanish is called the center variety of the family of polynomial differential systems. By the Hilbert Basis Theorem, the center variety is an algebraic set. Necessary conditions to have a center at the origin will be obtained by finding the zeros of the Liapunov constants.

We note that the space of systems (1) with a center at the origin is invariant with respect to the action group $C^{*}$ of change of variables $z \rightarrow \xi z$ :

$$
\begin{array}{ll}
A \rightarrow \xi^{(d-7) / 2} \bar{\xi}^{(d-5) / 2} \xi^{4+j} \bar{\xi}^{1-j} A, & B \rightarrow \xi^{(d-7) / 2} \bar{\xi}^{(d-5) / 2} \xi^{3} \bar{\xi}^{2} B, \\
C \rightarrow \xi^{(d-7) / 2} \bar{\xi}^{(d-5) / 2} \xi^{2-j} \bar{\xi}^{3+j} C, & D \rightarrow \xi^{(d-7) / 2} \bar{\xi}^{(d-5) / 2} \bar{\xi}^{5} D .
\end{array}
$$

To show the sufficiency of the found conditions either we will look for the existence of a local analytic first integral defined in a neighborhood of the origin, or we will show that system (1) is reversible. We recall that system (1) is reversible with respect to a straight line if it is invariant under the change of variables $(z, t) \rightarrow(w, \tau)$ where $\bar{w}=e^{i \gamma} z, \tau=-t$ for some $\gamma$ real. For system (1) we have the following result whose proof can be found in [8].

Lemma 4. System (1) is reversible if and only if $A=-\bar{A} e^{-2(1+j) i \gamma}, B=-\bar{B}$, $C=-\bar{C} e^{2(1+j) i \gamma}$ and $D=-\bar{D} e^{6 i \gamma}$ and for some $\gamma \in \mathbb{R}$. Furthermore in this situation the origin of system (1) is a center.

Once we have proven the existence of the so-called center variety of system (1) we also want to determine which of the centers are isochronous. In that case, let $z=0$ be a center (that is, we assume that we are under the hypothesis that guarantee that $z=0$ is a center) and let $V$ be a neighborhood of $z=0$ such that $V \backslash\{0\}$ is covered with cycles surrounding $z=0$. We can define a function, 
the period function of $z=0$ by associating to every point $z$ of $V$ the minimal period of the cycle passing through $z$. The center $z=0$ of system (1) is isochronous if the period of all integral curves in $V \backslash\{0\}$ is constant. The study of the isochronous centers started when Huygens which studied the cycloidal pendulum. This pendulum has isochronous oscillations in opposition to the monotonicity of the period for the usual pendulum. The existence of isochronous centers for several classes of systems has been studied in [5].

If we take the equation of $\theta^{\prime}=d \theta / d t$ we obtain

$$
\begin{aligned}
T & =\int_{0}^{2 \pi} \frac{d \theta}{\theta^{\prime}}=\int_{0}^{2 \pi} \frac{1}{1+G(\theta) r(\theta)^{d-1}} d \theta \\
& =\int_{0}^{2 \pi}(1-G(\theta) \rho(\theta)) d \theta=2 \pi-\int_{0}^{2 \pi} G(\theta) \rho(\theta) d \theta
\end{aligned}
$$

where $\rho(\theta)=\sum_{j \geq 1} \rho_{j}(\theta) \gamma^{j}$ is given in (5) and $\rho_{j}(\theta)$ are the terms giving rise to the Liapunov-Abel constants. Then system (1) has an isochronous center at the origin if it is a center and satisfies

$$
\int_{0}^{2 \pi} G(\theta) \varrho(\theta) d \theta=\sum_{j \geq 1}\left(\int_{0}^{2 \pi} G(\theta) \rho_{j}(\theta) d \theta\right) \gamma^{j}=0
$$

that is

$$
T=\int_{0}^{2 \pi} \frac{d \theta}{\theta^{\prime}}=2 \pi-\sum_{j \geq 1} T_{j} \gamma^{j}=2 \pi
$$

if and only if

$$
T_{j}=\int_{0}^{2 \pi} G(\theta) \rho_{j}(\theta) d \theta=0, \quad \text { for } j \geq 1 .
$$

The constants $T_{j}$ are called the period Abel constants.

\section{Proof of Theorem 1(a)}

We divide the proof of Theorem 1(a) into different parts.

\subsection{Sufficient conditions for a center}

In this ssection we will see that conditions (a.1), (a.2) and (a.3) are sufficient to have a center at the origin. For this we will prove that they have either a first integral defined in a neighborhood of zero (and consequently the origin cannot be a focus and must be a center), or they have the reversible symmetry described in Lemma 4.

Under conditions (a.1) if we rescale system (1) by $|z|^{d-5}$ it becomes

$$
\dot{z}=i z|z|^{5-d}+A z^{4+j} \bar{z}^{1-j}+i b_{2} z^{3} \bar{z}^{2}-\frac{(4+j) \bar{A}}{2-j} z^{2-j} \bar{z}^{3+j}+D \bar{z}^{5}=i \frac{\partial H}{\partial \bar{z}},
$$


where for $d \geq 5$ odd with $d \neq 7$ we have

$H=\frac{2}{7-d}|z|^{7-d}-i \frac{A}{2-j} z^{4+j} \bar{z}^{2-j}+i \frac{\bar{A}}{2-j} z^{2-j} \bar{z}^{4+j}+\frac{b_{2}}{3} z^{3} \bar{z}^{3}-\frac{i}{6}\left(D \bar{z}^{6}-\bar{D} z^{6}\right)$,

and for $d=7$ we have

$$
H=\log |z|^{2}-i \frac{A}{2-j} z^{4+j} \bar{z}^{2-j}+i \frac{\bar{A}}{2-j} z^{2-j} \bar{z}^{4+j}+\frac{b_{2}}{3} z^{3} \bar{z}^{3}-\frac{i}{6}\left(D \bar{z}^{6}-\bar{D} z^{6}\right) .
$$

Note that the integrals $\exp (H)$ for $d=7$ and $H$ for $d \geq 5$ odd with $d \neq 7$, are real and well defined at the origin. Therefore the origin is a Hamiltonian center.

From the conditions (a.2) and (a.3), we obtain

$$
B=-\bar{B}, \quad \frac{\bar{A}}{A}=\frac{C}{\bar{C}}, \quad\left(\frac{\bar{A}}{A}\right)^{3}=-\left(-\frac{D}{\bar{D}}\right)^{j+1}, \quad\left(\frac{\bar{C}}{C}\right)^{3}=-\left(\frac{-\bar{D}}{D}\right)^{j+1} .
$$

Now let $\theta_{1}, \theta_{2}$ and $\theta_{3}$ such that $e^{i \theta_{1}}=-\bar{A} / A, e^{i \theta_{2}}=-\bar{C} / C$ and $e^{i \theta_{3}}=(-\bar{D} / D)^{j+1}$. Then by (9) we obtain

$$
\theta_{1}=-\theta_{2}(\bmod .2 \pi) \text { and } 3 \theta_{2}=\theta_{3}(\bmod .2 \pi) .
$$

Now taking $\gamma=\theta_{1} /(2(1+j))$ and using (10) we have

and

$$
e^{-2(1+j) i \gamma}=e^{-i \theta_{1}}=-\frac{A}{\bar{A}}, \quad e^{2(1+j) i \gamma}=e^{i \theta_{1}}=e^{-i \theta_{2}}=-\frac{C}{\bar{C}},
$$

$$
e^{6 i \gamma}=e^{3 i \theta_{1} /(1+j)}=e^{-3 i \theta_{2} /(1+j)}=e^{-i \theta_{3} /(1+j)}=-\frac{D}{\bar{D}} .
$$

By Lemma 4 these imply that system (1) under conditions (a.2) and (a.3) is reversible and consequently has a center at the origin.

\subsection{Necessary conditions for a center}

In this section we will see that conditions (a.1), (a.2) and (a.3) are necessary to have a center at the origin. For this we will first compute the Liapunov constants up to some order and then show that the zeros of those Liapunov constants are precisely conditions (a.1), (a.2) or (a.3).

Proposition 5. The Liapunov constants of system (1) when $j=0$ with $d \geq 5$ odd are

$$
\begin{aligned}
V_{1}= & e^{2 \pi(d-1) \lambda} \\
V_{2}= & b_{1}, \\
V_{3}= & -\operatorname{Im}(A C), \\
V_{4}= & 0, \\
V_{5}= & \operatorname{Im}((2 A+\bar{C}) D[(d-3) A+(d+1) \bar{C}][(d-7) A+(d+5) \bar{C}]), \\
V_{6}= & \operatorname{Re}\left(( 2 A + \overline { C } ) B D \left[\left(4 d^{2}-29 d+49\right) A^{2}+\left(4 d^{2}+2 d-54\right) A \bar{C}\right.\right. \\
& \left.\left.+(11 d+13) \bar{C}^{2}\right]\right),
\end{aligned}
$$


and

$$
V_{7}=\operatorname{Im}\left((2 A+\bar{C}) D\left[A^{2} u+\bar{C}^{2} v\right]\right)
$$

with

$$
u=288\left(5|A|^{2}-6|D|^{2}\right), \quad v=-5760|A|^{2}+4320|D|^{2} \quad \text { if } d=7,
$$

and if $d \neq 7$ odd then

$$
\begin{aligned}
u= & \left(8,640-31,680 d+44,160 d^{2}-28,800 d^{3}+8,640 d^{4}-960 d^{5}\right)|A|^{2} \\
& -\left(41,910-48,248 d+29 d^{2}+6,541 d^{3}+64 d^{4}-278 d^{5}-19 d^{6}+d^{7}\right)|D|^{2}
\end{aligned}
$$

and

$$
\begin{aligned}
v= & \left(-960+960 d+1,920 d^{2}-1,920 d^{3}-960 d^{4}+960 d^{5}\right)|A|^{2} \\
& +\left(4,950+6,680 d-1,947 d^{2}-6,547 d^{3}-2,736 d^{4}-390 d^{5}-11 d^{6}+d^{7}\right)|D|^{2} .
\end{aligned}
$$

Furthermore $V_{8}=0$ and

$$
V_{9}=\rho_{9}(2 \pi)=\operatorname{Im}\left((2 A+\bar{C}) D\left(A^{2} u_{1}+\bar{C}^{2} v_{1}\right)\right)
$$

where

$$
v_{1}= \begin{cases}-9 u_{1}, & u_{1}=|C|^{4} \\ -5 u_{1}, & \text { if } d=5 \\ u_{1}=|D|^{4} & \text { if } d \neq 5 .\end{cases}
$$

We remark that $V_{k} \equiv \rho_{k}(2 \pi)\left(\bmod .\left\{V_{1}, V_{2}, \ldots, V_{k-1}\right\}\right)$, for $k=1, \ldots, 9$ and also modulo a positive constant.

Proof. Solving $\rho_{1}^{\prime}(\theta)=(d-1) \lambda \rho_{1}(\theta)$ and evaluating at $\theta=2 \pi$ we obtain $v_{1}=$ $\rho_{1}(2 \pi)=e^{2 \pi(d-1) \lambda}$. Then $V_{1}=e^{2 \pi(d-1) \lambda}$. In what follows we take $\lambda=0$.

Substituting (5) into (4) we get that the functions $\rho_{k}(\theta)$ must satisfy

$$
\begin{aligned}
\rho_{2}^{\prime}= & G \rho_{1}^{2}, \\
\rho_{3}^{\prime}= & F \rho_{1}^{3}+2 G \rho_{1} \rho_{2}, \\
\rho_{4}^{\prime}= & 3 F \rho_{1}^{2} \rho_{2}+G\left(\rho_{2}^{2}+2 \rho_{1} \rho_{3}\right), \\
\rho_{5}^{\prime}= & 3 F\left(\rho_{1} \rho_{2}^{2}+\rho_{1}^{2} \rho_{3}\right)+2 G\left(\rho_{2} \rho_{3}+\rho_{1} \rho_{4}\right), \\
\rho_{6}^{\prime}= & F\left(\rho_{2}^{3}+6 \rho_{1} \rho_{2} \rho_{3}+3 \rho_{1}^{2} \rho_{4}\right)+G\left(\rho_{3}^{2}+2 \rho_{2} \rho_{4}+2 \rho_{1} \rho_{5}\right), \\
\rho_{7}^{\prime}= & 3 F\left(\rho_{2}^{2} \rho_{3}+\rho_{1} \rho_{3}^{2}+2 \rho_{1} \rho_{2} \rho_{4}+\rho_{1}^{2} \rho_{5}\right)+2 G\left(\rho_{3} \rho_{4}+\rho_{2} \rho_{5}+\rho_{1} \rho_{6}\right), \\
\rho_{8}^{\prime}= & 3 F\left(\rho_{2} \rho_{3}^{2}+\rho_{2}^{2} \rho_{4}+2 \rho_{1} \rho_{3} \rho_{4}+2 \rho_{1} \rho_{2} \rho_{5}+\rho_{1}^{2} \rho_{6}\right) \\
& +G\left(\rho_{4}^{2}+2 \rho_{3} \rho_{5}+2 \rho_{2} \rho_{6}+2 \rho_{1} \rho_{7}\right), \\
\rho_{9}^{\prime}= & F\left(\rho_{3}^{3}+\rho_{2} \rho_{3} \rho_{4}+3 \rho_{1} \rho_{4}^{2}+3 \rho_{2}^{2} \rho_{5}+6 \rho_{1} \rho_{3} \rho_{5}+6 \rho_{1} \rho_{2} \rho_{6}+3 \rho_{1}^{2} \rho_{7}\right) \\
& +2 G\left(\rho_{4} \rho_{5}+\rho_{3} \rho_{6}+\rho_{2} \rho_{7}+\rho_{1} \rho_{8}\right) .
\end{aligned}
$$

where we have omitted that all the functions depend on $\theta$. Note that all these differential equations can be solved recursively doing a integral between 0 and $\theta$, and recalling that $\rho_{k}(0)=0$ for $k \geq 2$. We have done all the computations of this paper 
with the help of the algebraic manipulator mathematica. These computations are not difficult but are long and tedious.

Solving the equation $\rho_{2}^{\prime}=G \rho_{1}^{2}$ we get that $\rho_{2}(2 \pi)=2 \pi(d-1) b_{1}$. Then $V_{2}=b_{1}$. From now on we take $b_{1}=0$.

Now we compute the solution $\rho_{3}(\theta)$ of $\rho_{3}^{\prime}=F \rho_{1}^{3}+2 G \rho_{1} \rho_{2}$, and we get that $\rho_{3}(2 \pi)=2 \pi(1-d) \operatorname{Im}(A C)$. Then $V_{3}=-\operatorname{Im}(A C)$.

Computing the solution $\rho_{4}(\theta)$ from the differential equation for $\rho_{4}(\theta)$, we get $\rho_{4}(\theta)$ and in particular we obtain that $V_{4}=0$, being $V_{4}$ equal to $\rho_{4}(2 \pi)$ when $\rho_{2}(2 \pi)=\rho_{3}(2 \pi)=0$.

Solving the differential equation for $\rho_{5}(\theta)$ we get $\rho_{5}(\theta)$ and in particular we obtain from the expression of $v_{5}=\rho_{5}(2 \pi)$ the value of $V_{5}$ given in the statement of Proposition 5 modulo $\rho_{2}(2 \pi)=\rho_{3}(2 \pi)=0$ and a positive constant. More precisely we can check that if we multiply $v_{5}$ by $24 /(\pi(d-1))$ then

$$
\begin{aligned}
v_{5}= & V_{5}-V_{3}\left(\left(-46-36 d+10 d^{2}\right) a_{2} d_{2}+\left(12-8 d-4 d^{2}\right) c_{2} d_{2}\right. \\
& \left.+\left(23+18 d-5 d^{2}\right) a_{1} d_{1}+\left(24-16 d-8 d^{2}\right)\left(c_{1} d_{1}\right)\right) .
\end{aligned}
$$

We compute the solution $\rho_{k}(\theta)$ for $k=6, \ldots, 9$ from the differential equation for $\rho_{k}(\theta)$, we get $\rho_{k}(\theta)$, and in particular we obtain the expression for $v_{k}=$ $\rho_{k}(2 \pi)$ given in the statement of Proposition 5 modulo $\rho_{2}(2 \pi)=\rho_{3}(2 \pi)=\cdots=$ $\rho_{k-1}(2 \pi)=0$ and a positive constant. The computation of $V_{k}$ for $k=6, \ldots, 9$ is done in the same way as $V_{5}$. This completes the proof of the proposition.

Proposition 6. Let $j=0$. For $d \geq 29$ odd if $V_{1}=1, V_{2}=V_{3}=V_{4}=V_{5}=V_{6}=$ $V_{7}=0$, then either (a.1), or (a.2) holds. Furthermore for any $d \in\{5,7, \ldots, 27\}$ if $V_{1}=1, V_{2}=V_{3}=V_{4}=V_{5}=V_{6}=V_{7}=V_{8}=V_{9}=0$, then either (a.1), or (a.2) holds.

Proof. We do the proof of the two statements simultaneously. From the fact that $V_{1}=1$ we get that $\lambda=0$. Furthermore to make $V_{3}=0$ we will consider two different cases: $C=0$ and $C \neq 0$. In this last case we have that $A=\mu \bar{C}$ with $\mu \in \mathbb{R}$.

Case 1: $C=0$. In this case

$$
V_{5}=2(d-3)(d-7) \operatorname{Im}\left(A^{3} D\right) .
$$

In view of the factors of $V_{5}$ and since $d \geq 5$ odd, we need to consider two different subcases.

Subcase 1.1: $\operatorname{Im}\left(A^{3} D\right)=0$. Therefore we are under the hypotheses of condition (a.2).

Subcase 1.2: $\operatorname{Im}\left(A^{3} D\right) \neq 0$ and $d=7$. Since $b_{1}=0$ we have

$$
V_{6}=84 \operatorname{Re}\left(A^{3} B D\right)=-84 b_{2} \operatorname{Im}\left(A^{3} D\right) .
$$

To have $V_{6}=0$ we must impose $b_{2}=0$, that is $B=0$. Then

$$
V_{7}=576\left(5|A|^{2}-6|D|^{2}\right) \operatorname{Im}\left(A^{3} D\right) \text {. }
$$


In order to have $V_{7}=0$ we must impose $5|A|^{2}=6|D|^{2}$. Then $V_{7}=V_{8}=0$ and

$$
V_{9}=2|D|^{4} \operatorname{Im}\left(A^{3} D\right) .
$$

Since $\operatorname{Im}\left(A^{3} D\right) \neq 0$ (and thus in particular $D \neq 0$ ) we have that $V_{9} \neq 0$ and this case does not yield any center.

Case 2: $A=\mu \bar{C}, \mu \in \mathbb{R}$. In this case

$$
V_{5}=(2 \mu+1)((d-3) \mu+(d+1))((d-7) \mu+(d+5)) \operatorname{Im}\left(\bar{C}^{3} D\right) .
$$

In view of the factors of $V_{5}$ we need to consider four different subcases.

Subcase 2.1: $\mu=-1 / 2$. Then we are under the hypotheses of condition (a.1).

Subcase 2.2: $\operatorname{Im}\left(\bar{C}^{3} D\right)=0$. Therefore we are under the hypotheses of condition (a.2).

Subcase 2.3: $\mu=-(d+1) /(d-3), \operatorname{Im}\left(\bar{C}^{3} D\right) \neq 0$. Since $b_{1}=0$, we have

$$
V_{6}=-\frac{4(d-1)^{3}(d+5)}{(d-3)^{3}} \operatorname{Re}\left(B \bar{C}^{3} D\right)=-\frac{4(d-1)^{3}(d+5)}{(d-3)^{3}} b_{2} \operatorname{Im}\left(\bar{C}^{3} D\right) .
$$

Then, since $d \geq 5$ odd, $V_{6}=0$ if and only if $b_{2}=0$, that is $B=0$. Computing $V_{7}$ we obtain

$$
V_{7}= \begin{cases}-7776|D|^{2}(d+1) /(d-3)-2 & \text { if } d=7 \\ 2(d+5)^{2} C_{d}|D|^{2} /(d-3)^{2} & \text { otherwise }\end{cases}
$$

where

$$
C_{d}=-2882-2548 d+3037 d^{2}+2975 d^{3}-264 d^{4}-262 d^{5}-19 d^{6}+d^{7} .
$$

Since for $d \geq 5$ odd, $C_{d} \neq 0$, we have that $D=0$, a contradiction with the fact that $\operatorname{Im}\left(\bar{C}^{3} D\right) \neq 0$, and thus this condition does not yield a center.

Subcase 2.4: $\mu=-(d+5) /(d-7), d \neq 7$ and $\operatorname{Im}\left(\bar{C}^{3} D\right) \neq 0$. Since $b_{1}=0$ we have

$$
V_{6}=-\frac{28(d+17)(d-1)^{3}}{(d-7)^{3}} \operatorname{Re}\left(B \bar{C}^{3} D\right)=-\frac{28(d+17)(d-1)^{3}}{(d-7)^{3}} b_{2} \operatorname{Im}\left(\bar{C}^{3} D\right) .
$$

Then, since $d \geq 5$ odd, $V_{6}=0$ if and only if $b_{2}=0$, that is $B=0$. Computing $V_{7}$ we obtain

$$
V_{7}=\frac{\left(d^{2}+16 d-17\right)}{(d-7)^{3}(d-3)^{2}} K_{d}(C, D)
$$

where

$$
\begin{aligned}
K_{d}(C, D)= & 7,680(d-1)^{3}(d+1)^{2}\left(d^{2}-2 d-11\right)|C|^{2} \\
& +\left(d^{2}+2 d-15\right)^{2}\left(d^{6}-26 d^{5}-96 d^{4}+776 d^{3}+245 d^{2}\right. \\
& -4,902 d-25,806)|D|^{2} .
\end{aligned}
$$

Since for $d \geq 5$ odd, $d^{2}+16 d-17 \neq 0$ and $d^{2}-2 d-11>0$, and for $d \geq 29$ odd the quantity $d^{6}-26 d^{5}-96 d^{4}+776 d^{3}+245 d^{2}-4,902 d-25,806$ is always positive, we have that for $d \geq 29$ odd in order to have $V_{7}=0$ we must impose $C=D=0$, 
a contradiction with the fact that $\operatorname{Im}\left(\bar{C}^{3} D\right) \neq 0$, and thus this condition does not yield a center.

On the other hand, for $d \in\{5,9, \ldots, 25,27\}$ odd, with $d \neq 7$, imposing $K_{d}(C, D)=0$, we have $V_{7}=0$. Then $V_{8}=0$ and

$$
V_{9}=\frac{4}{(d-7)^{3}}\left(d^{3}-3 d^{2}-285 d+935\right)|D|^{4} \operatorname{Im}\left(\bar{C}^{3} D\right) .
$$

Since when $d$ is an integer, the quantity $d^{3}-3 d^{2}-285 d+935$ is never zero, we have that $V_{9}=0$ if and only if $D=0$, a contradiction with the fact that $\operatorname{Im}\left(\bar{C}^{3} D\right) \neq 0$, and thus this condition does not yield a center. This completes the proof of Proposition 6 .

Proposition 7. The Liapunov constants of system (1) when $j=1$, with $d \geq 5$ odd are

$$
\begin{aligned}
V_{1}= & e^{2 \pi(d-1) \lambda} \\
V_{2}= & b_{1}, \\
V_{3}= & -\operatorname{Im}(A C), \\
V_{4}= & 0, \\
V_{5}= & 0, \\
V_{6}= & \operatorname{Re}\left((5 A+\bar{C}) D^{2}[(d-7) A+(d+5) \bar{C}][(d-17) A+(d+15) \bar{C}]\right), \\
V_{7}= & \operatorname{Im}\left(( 5 A + \overline { C } ) B D ^ { 2 } \left[\left(5 d^{2}-15 d-302\right) A^{2}+\left(-5 d^{2}+130 d+499\right) A \bar{C}\right.\right. \\
& \left.\left.-\left(10 d^{2}+95 d+207\right) \bar{C}^{2}\right]\right),
\end{aligned}
$$

and

$$
V_{8}=\operatorname{Re}\left((5 A+\bar{C}) D^{2}\left[A^{2} u+\bar{C}^{2} v\right]\right)
$$

where for $d=7$,

$$
u=\frac{11}{3584}\left(2,731|A|^{2}+544|D|^{2}\right), \quad v=-\frac{121}{25}\left(\frac{695,415}{681,472}|A|^{2}+\frac{1,535}{1,232}|D|^{2}\right),
$$

for $d=17$,

$$
u=-\frac{11}{63}\left(2,919|A|^{2}-2,068|D|^{2}\right), \quad v=\frac{121}{25}\left(\frac{260,785}{3,993}|A|^{2}+\frac{820}{9}|D|^{2}\right),
$$

and if $d \neq\{7,17\}$ odd then

$$
u=315(d-1)^{2} u_{1}|A|^{2}+2(d+5)^{2}(15+d)^{2} u_{2}|D|^{2},
$$

with

$$
\begin{aligned}
& u_{1}=329 d^{5}-3,025 d^{4}-21,654 d^{3}+144,334 d^{2}+1,202,429 d+1,976,915, \\
& u_{2}=43 d^{4}-1,600 d^{4}+10,260 d^{3}+61,470 d^{2}-345,295 d+351,154,
\end{aligned}
$$

and

$$
v=-315(d-1)^{2} v_{1}|A|^{2}-2(d+5)^{3}(15+d) v_{2}|D|^{2}
$$


with

$$
\begin{aligned}
& v_{1}=329 d^{5}+5,487 d^{4}+36,202 d^{3}+184,910 d^{2}+950,525 d+2,121,875, \\
& v_{2}=43 d^{4}+317 d^{3}+2,611 d^{2}+9,271 d+430 .
\end{aligned}
$$

Furthermore $V_{9}=0$ and

$$
V_{10}= \begin{cases}0 & \text { if } d=5, \\ \operatorname{Re}\left((5 A+\bar{C}) D^{2} A^{2}|D|^{4}\right) & \text { if } d=17, \\ \operatorname{Re}\left((5 A+\bar{C}) D^{2} \bar{C}^{2}|D|^{4}\right) & \text { if } d=7,9, \\ -\operatorname{Re}\left((5 A+\bar{C}) D^{2} \bar{C}^{2}|D|^{4}\right) & \text { if } d=11,13, \ldots, 39 .\end{cases}
$$

We remark that $V_{k} \equiv \rho_{k}(2 \pi)\left(\bmod .\left\{V_{1}, V_{2}, \ldots, V_{k-1}\right\}\right)$, for $k=1, \ldots, 10$ and also modulo a positive constant.

Proof. Proceeding as in the proof of Proposition 5 we readily obtain $V_{1}, V_{2}$ and $V_{3}$ as in the statement of that proposition. Now computing the solution $\rho_{k}(\theta)$ for $k=4,5$ from the differential equation for $\rho_{k}(\theta)$ (see $(11)$ ), we get $\rho_{k}(\theta)$ and in particular we obtain that $V_{k}=0$, being $V_{k}$ equal to $\rho_{k}(2 \pi)$ when $\rho_{2}(2 \pi)=\cdots=$ $\rho_{k-1}(2 \pi)=0$ for $k=4,5$.

We compute the solution $\rho_{6}(\theta)$ from the differential equation for $\rho_{6}(\theta)$, we get $\rho_{6}(\theta)$, and in particular we obtain the expression for $v_{6}=\rho_{6}(2 \pi)$ given in the statement of Proposition 5 modulo $\rho_{2}(2 \pi)=\rho_{3}(2 \pi)=0$ and a positive constant. More precisely we can check that if we multiply $v_{6}$ by $1536 /((d+1)(d+3) \pi)$ then

$$
\begin{aligned}
v_{6}= & V_{6}+V_{3}\left(\left(-1,662-88 d+22 d^{2}\right)\left(a_{2} d_{2}^{2}-a_{1} d_{1} d_{2}-a_{2} d_{1}^{2}\right)\right. \\
& \left.-\left(185+96 d+7 d^{2}\right)\left(c_{2} d_{2}^{2}-c_{2} d_{1}^{2}+4 c_{1} d_{1} d_{2}\right)\right) .
\end{aligned}
$$

We compute the solution $\rho_{k}(\theta)$ for $k=7,8,9,10$ from the differential equation for $\rho_{k}(\theta)$ and also using

$$
\begin{aligned}
\rho_{10}^{\prime}= & 3 F\left(\rho_{3}^{2} \rho_{4}+\rho_{2} \rho_{4}^{2}+2 \rho_{2} \rho_{3} \rho_{5}+2 \rho_{1} \rho_{4} \rho_{5}+\rho_{2}^{2} \rho_{6}+2 \rho_{1} \rho_{3} \rho_{6}+2 \rho_{1} \rho_{2} \rho_{7}\right. \\
& \left.+\rho_{1}^{2} \rho_{8}\right)+G\left(\rho_{5}^{2}+2 \rho_{4} \rho_{6}+2 \rho_{3} \rho_{7}+2 \rho_{2} \rho_{8}+2 \rho_{1} \rho_{9}\right) .
\end{aligned}
$$

We get $\rho_{k}(\theta)$ and in particular we obtain the expression for $v_{k}=\rho_{k}(2 \pi)$ given in the statement of Proposition 5 modulo $\rho_{2}(2 \pi)=\rho_{3}(2 \pi)=\rho_{k-1}(2 \pi)=0$ and a positive constant. The computation of $V_{k}$ is done in the same way as $V_{6}$. This completes the proof of the proposition.

We introduce a new condition

(a.4) $\lambda=b_{1}=3 A-5 \bar{C}=16|C|^{2}-9|D|^{2}=0$ and $d=5$ and $j=1$.

Proposition 8. Let $j=1$. For $d \geq 41$ odd if $V_{1}=1, V_{2}=V_{3}=V_{4}=V_{5}=V_{6}=$ $V_{7}=V_{8}=0$, then either (a.1) or (a.3) holds. Furthermore for any $d \in\{7, \ldots, 39\}$ if $V_{1}=1, V_{2}=V_{3}=V_{4}=V_{5}=V_{6}=V_{7}=V_{8}=V_{9}=V_{10}=0$, then either (a.1) or (a.3) holds. Moreover, if $d=5$ then if $V_{1}=1, V_{2}=V_{3}=V_{4}=V_{5}=V_{6}=V_{7}=$ $V_{8}=V_{9}=V_{10}=0$, then either (a.1), or (a.3), or (a.4) holds. 
Proof. We do the proof of the two propositions simultaneously. From the fact that $V_{1}=1$ we get that $\lambda=0$. Furthermore to make $V_{3}=0$ we will consider two different cases: $C=0$ and $C \neq 0$. In this last case we have that $A=\mu \bar{C}$, with $\mu \in \mathbb{R}$.

Case 1: $C=0$. In this case

$$
V_{5}=5(d-7)(d-17) \operatorname{Re}\left(A^{3} D^{2}\right) .
$$

In view of the factors of $V_{5}$ and since $d \geq 5$ odd, we need to consider three different subcases.

Subcase 1.1: $\operatorname{Re}\left(A^{3} D^{2}\right)=0$. Then we are under the hypotheses of condition (a.3).

Subcase 1.2: $\operatorname{Re}\left(A^{3} D^{2}\right) \neq 0$ and $d=7$. Since $b_{1}=0$, we have

$$
V_{7}=-162 b_{2} \operatorname{Re}\left(A^{3} D^{2}\right) .
$$

To have $V_{7}=0$ we must impose $b_{2}=0$, that is $B=0$. Then

$$
V_{8}=\frac{55}{3584}\left(2731|A|^{2}+514|D|^{2}\right) \operatorname{Re}\left(A^{3} D^{2}\right) .
$$

Since $\operatorname{Re}\left(A^{3} D^{2}\right) \neq 0$ we have that $V_{8} \neq 0$ and thus this case does not yield any center.

Subcase 1.3: $\operatorname{Re}\left(A^{3} D^{2}\right) \neq 0$ and $d=17$. Since $b_{1}=0$, we have

$$
V_{7}=888 b_{2} \operatorname{Re}\left(A^{3} D^{2}\right) \text {. }
$$

To have $V_{7}=0$ we must impose $b_{2}=0$, that is $B=0$. Then

$$
V_{8}=-\frac{55}{63}\left(2919|A|^{2}-2068|D|^{2}\right) \operatorname{Re}\left(A^{3} D^{2}\right) .
$$

Since $\operatorname{Re}\left(A^{3} D^{2}\right) \neq 0$ in order to have $V_{8}=0$ we must impose $2919|A|^{2}=2068|D|^{2}$. Then $V_{8}=V_{9}=0$ and

$$
V_{10}=5|D|^{4} \operatorname{Re}\left(A^{2} D^{2}\right) .
$$

In order to have $V_{10}=0$ we must impose $D=0$ but then $\operatorname{Re}\left(A^{3} D^{2}\right)=0$, a contradiction. Hence this case does not yield any center.

Case 2: $A=\mu \bar{C}, \mu \in \mathbb{R}$. In this case

$$
V_{6}=(5 \mu+1)((d-7) \mu+(d+5))((d-17) \mu+(d+15)) \operatorname{Re}\left(\bar{C}^{3} D^{2}\right) .
$$

In view of the factors in $V_{6}$ we need to consider four different subcases.

Subcase 2.1: $\mu=-1 / 5$. Then we are under the hypotheses of condition (a.1).

Subcase 2.2: $\operatorname{Re}\left(\bar{C}^{3} D^{2}\right)=0$. Therefore we are under the hypotheses of condition (a.3).

Subcase 2.3: $\mu=-(d+5) /(d-7), \operatorname{Re}\left(\bar{C}^{3} D^{2}\right) \neq 0$ and $d \neq 7$. Since $b_{1}=0$, we have

$$
V_{7}=-\frac{8\left(140,632+60,379 d+2,470 d^{2}-1,240 d^{3}-70 d^{4}+5 d^{5}\right)}{(d-7)^{3}} b_{2} \operatorname{Re}\left(\bar{C}^{3} D^{2}\right) .
$$


Since $d \geq 5$ odd, $V_{7}=0$ if and only if $b_{2}=0$, that is $B=0$. Computing $V_{8}$ we obtain

$$
\begin{aligned}
V_{8}= & -\frac{11(d-1)^{3}(d+8) \pi}{6,048(d-7)^{5}} \operatorname{Re}\left(\bar{C}^{3} D^{2}\right) \\
& \times\left((d+5)\left(5 d^{2}+6 d-83\right)(d-7)^{2}|D|^{2}+63(d-1)^{2}(7 d+29)|C|^{2}\right) .
\end{aligned}
$$

Since $d \geq 5$ we have that $V_{8}$ cannot vanish. So in this subcase we do not have a center at the origin.

Subcase 2.4: $\mu=-(d+15) /(d-17), \operatorname{Re}\left(\bar{C}^{3} D^{2}\right) \neq 0$ and $d \neq 17$. Since $b_{1}=0$, we have

$$
V_{7}=-\frac{8\left(2,932,707+898,009 d+10,270 d^{2}-6,990 d^{3}-145 d^{4}+5 d^{5}\right)}{(d-17)^{3}} b_{2} \operatorname{Re}\left(\bar{C}^{3} D^{2}\right) \text {. }
$$

Since $d \geq 5$ odd, $V_{7}=0$ if and only if $b_{2}=0$, that is $B=0$. Computing $V_{8}$ we obtain

$$
\begin{aligned}
V_{8}= & \frac{(d-1)^{3}(d+23) \pi}{6,048(d-17)^{5}} \operatorname{Re}\left(\bar{C}^{3} D^{2}\right)\left(2,016(d-1)^{2}(49 d-277)|C|^{2}\right. \\
& \left.+\left(31 d^{3}-1,237 d^{2}-2,035 d+41,257\right)(d-17)^{2}|D|^{2}\right) .
\end{aligned}
$$

Therefore if $V_{8}=0$, then $d \in\{5,7, \ldots, 39\}$. Moreover if $d \geq 41$ odd, $V_{8} \neq 0$ and thus it does not yield a center.

If $d \in\{5,7, \ldots, 39\}$ and $V_{8}=0$, then a computation shows that $V_{9}=0$.

When $d=5, V_{8}=0$ becomes $16|C|^{2}-9|D|^{2}=0$. Computing $V_{10}$ when $d=5$ we get that $V_{10}=0$ and we are under the assumptions (a.4).

When $d \in\{7,9, \ldots, 39\}$ computing $V_{10}$, after modulo a positive constant we have

$$
V_{10}= \begin{cases}|D|^{4} \operatorname{Re}\left(\bar{C}^{3} D^{2}\right) & \text { if } d=7,9 \\ -|D|^{4} \operatorname{Re}\left(\bar{C}^{3} D^{2}\right) & \text { if } d=11,13, \ldots, 39 .\end{cases}
$$

Since $D \neq 0$ (otherwise $\operatorname{Re}\left(\bar{C}^{3} D^{2}\right)=0$ we get that $V_{10} \neq 0$ and this condition does not yield a center. This completes the proof of Proposition 8 .

Proposition 9. Condition (a.4) does not provide a center at the origin.

Proof. Making the change $z \rightarrow w=\xi z$ with $\xi=\bar{C}^{-1 / 4}$ to system (1) and using (6) we obtain that system (1) under the assumptions (a.4), i.e. with $d=5$, $\lambda=B=3 A-5 \bar{C}=16|C|^{2}-9|D|^{2}=0$ and $\operatorname{Re}\left(\bar{C}^{3} D^{2}\right) \neq 0$ can be written as

$$
\dot{w}=i w+\left(\frac{5}{3} w^{5}+w \bar{w}^{4}+\frac{4}{3} e^{i \theta} \bar{w}^{5}\right),
$$

with $\sin \theta \neq 0$, i.e. $\theta \in(0,2 \pi) \backslash\{\pi\}$. For system (12) (in view of Proposition 8) we have that $V_{2}=\cdots=V_{10}=0$. Now using $\rho_{1}, \ldots, \rho_{10}$ computed in the proof of Proposition 7 and using that 


$$
\begin{aligned}
\rho_{11}^{\prime}= & 3 F\left(\rho_{3} \rho_{4}^{2}+\rho_{3}^{2} \rho_{5}+2 \rho_{2} \rho_{4} \rho_{5}+\rho_{1} \rho_{5}^{2}+2 \rho_{2} \rho_{3} \rho_{6}+2 \rho_{1} \rho_{4} \rho_{6}\right. \\
& \left.+\rho_{2}^{2} \rho_{7}+2 \rho_{1} \rho_{3} \rho_{7}+2 \rho_{1} \rho_{2} \rho_{8}+\rho_{1}^{2} \rho_{9}\right)+2 G\left(\rho_{1} \rho_{10}+\rho_{5} \rho_{6}\right. \\
& \left.+\rho_{4} \rho_{7}+\rho_{3} \rho_{8}+\rho_{2} \rho_{9}\right), \\
\rho_{12}^{\prime}= & -F\left(-3 \rho_{1}^{2} \rho_{10}-\rho_{4}^{3}-6 \rho_{3} \rho_{4} \rho_{5}-3 \rho_{2} \rho_{5}^{2}-3 \rho_{3}^{2} \rho_{6}-6 \rho_{2} \rho_{4} \rho_{6}\right. \\
& \left.-6 \rho_{1} \rho_{5} \rho_{6}-6 \rho_{2} \rho_{3} \rho_{7}-6 \rho_{1} \rho_{4} \rho_{7}-3 \rho_{2}^{2} \rho_{8}-6 \rho_{1} \rho_{3} \rho_{8}-6 \rho_{1} \rho_{2} \rho_{9}\right) \\
& -G\left(-2 \rho_{1} \rho_{11}-2 \rho_{10} \rho_{2}-\rho_{6}^{2}-2 \rho_{5} \rho_{7}-2 \rho_{4} \rho_{8}-2 \rho_{3} \rho_{9}\right), \\
\rho_{13}^{\prime}= & 3 F\left(\rho_{1}^{2} \rho_{11}+2 \rho_{1} \rho_{10} \rho_{2}+\rho_{4}^{2} \rho_{5}+\rho_{3} \rho_{5}^{2}+2 \rho_{3} \rho_{4} \rho_{6}+2 \rho_{2} \rho_{5} \rho_{6}\right. \\
& +\rho_{1} \rho_{6}^{2}+\rho_{3}^{2} \rho_{7}+2 \rho_{2} \rho_{4} \rho_{7}+2 \rho_{1} \rho_{5} \rho_{7}+2 \rho_{2} \rho_{3} \rho_{8}+2 \rho_{1} \rho_{4} \rho_{8} \\
& \left.+\rho_{2}^{2} \rho_{9}+2 \rho_{1} \rho_{3} \rho_{9}\right)+2 G\left(\rho_{1} \rho_{12}+\rho_{11} \rho_{2}+\rho_{10} \rho_{3}+\rho_{6} \rho_{7}+\rho_{5} \rho_{8}+\rho_{4} \rho_{9}\right) .
\end{aligned}
$$

we get that $V_{11}=V_{12}=0$ and $V_{13}=\sin \theta$, where $V_{k} \equiv \rho_{k}(2 \pi)$ modulo $\left\{V_{1}, V_{2}, \ldots\right.$, $\left.V_{k-1}\right\}$ ) for $k=11,12,13$, and also modulo a positive constant. Since $\sin \theta \neq 0$, we get that $V_{13} \neq 0$. This implies that system (12) does not have a center at the origin, and consequently condition (a.4) does not provide a center.

\section{Proof of Theorem $1(b)$}

We divide the proof of Theorem 1(b) into different parts.

\subsection{Sufficient conditions for an isochronous center}

In this subsection we will see that conditions (b.1), (b.2) and (b.3) are sufficient to have an isochronous center. For this we will prove that under conditions (b.1) or (b.2) or (b.3) Eq. (7) holds.

Since in assumptions (b.1) or (b.2) of (b.3), we can assume that $A \neq 0$ (otherwise we will obtain the linear center), we can make the change of variables

$$
\omega=\xi z \quad \text { where } \xi=\left(\frac{A^{d+1+2 j}}{\bar{A}^{d-3-2 j}}\right)^{1 /(4(d-1)(1+j))},
$$

and system (1) with hypothesis (b.1) becomes

$$
z^{\prime}=i z+(z \bar{z})^{(d-5) / 2}\left(z^{4+j} \bar{z}^{1-j}+z^{2-j} \bar{z}^{3+j}\right) .
$$

Rewriting (14) in polar coordinates we obtain

$$
r^{\prime}=2 r^{d} \cos 2 \theta, \quad \theta^{\prime}=1
$$

and clearly (7) holds.

Now system (1) with hypothesis (b.2) becomes

$$
z^{\prime}=i z+(z \bar{z})^{(d-5) / 2}\left(z^{4+j} \bar{z}^{1-j}-\frac{d-3-2 j}{d+1+2 j} z^{2-j} \bar{z}^{3+j}\right) .
$$


In polar coordinates it has the form

$$
r^{\prime}=\frac{4(1+j) r^{d}}{d+1+2 j} \cos (2(1+j) \theta), \quad \theta^{\prime}=1+\frac{2(d-1)}{d+1+2 j} r^{d-1} \sin (2(1+j) \theta) .
$$

Therefore

$$
\frac{d r}{d \theta}=\frac{4(1+j) r^{d} \cos (2(1+j) \theta)}{d+1+2 j+2(d-1) r^{d-1} \sin (2(1+j) \theta)} \quad \text { with } r(0)=r_{0} .
$$

Then integrating it and since $r(\theta) \geq 0$ for any $\theta$ we get that $r(\theta)^{1-d}$ is equal to

$$
\frac{-2(d-1) \sin (2(1+j) \theta)+\sqrt{(d+1+2 j)^{2} r_{0}^{2-2 d}+4(d-1)^{2} \sin ^{2}(2(1+j) \theta)}}{d+1+2 j} .
$$

Note that

$$
\sqrt{(d+1+2 j)^{2} r_{0}^{2-2 d}+4(d-1)^{2} \sin ^{2}(2(1+j) \theta)}>|2(d-1) \sin (2(1+j) \theta)|,
$$

and thus $r(\theta)$ given in (16) is positive. Therefore, introducing (16) into (15) we have that

$$
\begin{aligned}
\int_{0}^{2 \pi} \frac{d \theta}{\theta^{\prime}} & =\int_{0}^{2 \pi}\left(1-\frac{2(d-1) \sin (2(1+j) \theta)}{\sqrt{4(d-1)^{2} \sin ^{2}(2(1+j) \theta)+(d+1+2 j)^{2} r_{0}^{2-2 d}}}\right) d \theta \\
& =2 \pi,
\end{aligned}
$$

since the function

$$
2(d-1) \sin (2(1+j) \theta) / \sqrt{4(d-1)^{2} \sin ^{2}(2(1+j) \theta)+(d+1+2 j)^{2} r_{0}^{2-2 d}}
$$

is odd in $\theta$.

Now system (1) with hypothesis (b.3) becomes

$$
z^{\prime}=i z+(z \bar{z}) \bar{z}\left(z^{4}-\bar{z}^{4}\right) .
$$

In polar coordinates Eq. (17) has the form

$$
r^{\prime}=r^{7}(\cos (2 \theta)-\cos (6 \theta)), \quad \theta^{\prime}=1+r^{6}(\sin (2 \theta)+\sin (6 \theta)) .
$$

Note that system (18) has an invariant of the form

$$
I=I(t, r, \theta)=6 \theta+4 r^{6} \cos ^{3}(2 \theta)-6 t .
$$

Here $I$ is invariant in the sense that $d I / d t$ is zero on the solutions of system (18). From this invariant we can express the time in function of the variables $(r, \theta, I)$ as follows

$$
t=\theta+\frac{2}{3} r^{6} \cos ^{3}(2 \theta)-\frac{I}{6} .
$$


Let $r(\theta)$ be a solution of system (18) sufficiently close to the origin. Since the origin is a center, we get that $r(2 \pi)=r(0)$ in a certain neighborhood of the origin. Thus

$$
T=t(2 \pi)-t(0)=\left[2 \pi+\frac{2}{3} r(2 \pi)^{6}-\frac{I}{6}\right]-\left[\frac{2}{3} r(0)^{6}-\frac{I}{6}\right]=2 \pi .
$$

This completes the sufficiency of the conditions (b.1), (b.2) and (b.3).

\subsection{Necessary conditions for an isochronous center}

In this subsection we will see that conditions (b.1) or (b.2) or (b.3) are necessary to have an isochronous center. For this we will first compute the period constants up to some order and then show that the zeros of those period constants are precisely conditions (b.1) or (b.2) or (b.3).

We note that since $\rho_{1}(\theta)=1$, then from (3) and (8) we have $T_{1}=2 \pi b_{2}$. Then since by either condition (a.1), or (a.2) we have $b_{1}=0$, from now on we will assume that $B=0$.

Now we compute $T_{2}$ using $\rho_{2}(\theta)$ computed in Proposition 5, and Eqs. (3) and $(8)$. We get that after dividing by $\pi /(6(1+j))$ it is equal to

$T_{2}=3(d-3-2 j)|A|^{2}+12(1+j) \operatorname{Re}(A C)-3(d+1+2 j)|C|^{2}-(1+j)(d+5)|D|^{2}$.

We distinguish two different cases.

Case 1: $A=0$. In this case $T_{2}$ becomes

$$
T_{2}=-\frac{\pi}{6(1+j)}\left(3(d+1+2 j)|C|^{2}+(1+j)(d+5)|D|^{2}\right) .
$$

In order that $T_{2}=0$ we must impose $C=D=0$. Then $A=B=C=0$, which is not possible otherwise we have the linear center. Therefore this case does not provide an isochronous center.

Case 2: $A \neq 0$. In this case since from $V_{2}=0$ we have that $\operatorname{Im}(A C)=0$ we get that $C=\mu \bar{A}$ with $\mu \in \mathbb{R}$. We will consider two different subcases.

Subcase 2.1: $\mu=-(4+j) /(2-j)$. In this case $C=-(4+j) \bar{A} /(2-j)$ and we are under the hypothesis (c.1). Then $T_{2}$ becomes

$$
T_{2}=-\frac{\pi(d+5)}{6}\left(|D|^{2}+9(1+j)^{2}|A|^{2}\right) .
$$

Since $A \neq 0$, we get that $T_{2} \neq 0$. Therefore this case does not provide an isochronous center.

Subcase 2.2: $\mu \in \mathbb{R} \backslash\{-(4+j) /(2-j)\}$. In this case $C=\mu \bar{A}$ and we are in hypothesis (a.2) or (a.3). We consider two subcases.

Subcase 2.2.1: $j=0$. By the change of variables (13) we can rewrite system (1) as

$$
w^{\prime}=i w+(w \bar{w})^{(d-5) / 2}\left[w^{4} \bar{w}+\mu w^{2} \bar{w}^{3}+\tilde{D} \bar{w}^{5}\right]
$$


Since we are in assumptions (a.2) we must have $\tilde{d}_{2}=0$. Computing $T_{2}$ under these assumptions we have

$$
T_{2}=3(d-3)+12 \mu-3(d+1) \mu^{2}-(d+5) \tilde{d}_{1}^{2},
$$

modulo a non-zero constant. From now on we write $d_{1}$ instead of $\tilde{d}_{1}$.

We compute $T_{3}$ using $\rho_{3}(\theta)$ given in Proposition 5, (3) and (8), and we get that $T_{3}=0$. Then we compute $T_{4}$ using $\rho_{4}(\theta)$ given in Proposition $5,(3)$ and $(8)$. We get

$$
\begin{aligned}
T_{4}= & 6 d^{3} \mu^{4}+36 d^{2} \mu^{4}-6 d \mu^{4}-36 \mu^{4}+9 d^{3} \mu^{3}-57 d^{2} \mu^{3}-201 d \mu^{3} \\
& +3 d^{3} d_{1} \mu^{3}+19 d^{2} d_{1} \mu^{3}+21 d d_{1} \mu^{3}+5 d_{1} \mu^{3}+105 \mu^{3}-3 d^{3} \mu^{2} \\
& -87 d^{2} \mu^{2}-72 d_{1}^{2} \mu^{2}+471 d \mu^{2}+6 d^{3} d_{1} \mu^{2}-14 d^{2} d_{1} \mu^{2}-94 d_{1} \mu^{2} \\
& -42 d_{1} \mu^{2}+267 \mu^{2}-9 d^{3} \mu+57 d^{2} \mu+81 d \mu+3 d^{3} d_{1} \mu-37 d^{2} d_{1} \mu \\
& +69 d d_{1} \mu+109 d_{1} \mu-993 \mu-3 d^{3}+51 d^{2}+288 d_{1}^{2}-345 d-4 d^{2} d_{1} \\
& +40 d d_{1}-84 d_{1}+657 .
\end{aligned}
$$

In a similar way we get that $T_{5}=0$ and

$$
\begin{aligned}
T_{6}= & 25,200 d^{5} \mu^{6}+170,100 d^{4} \mu^{6}+169,032 d^{3} \mu^{6}-2,723,724 d^{2} \mu^{6} \\
& -2,717,544 d \mu^{6}+30,312 \mu^{6}+66,150 d^{5} \mu^{5}-626,328 d^{4} \mu^{5} \\
& -2,606,616 d^{3} \mu^{5}-3,813,360 d^{2} \mu^{5}+38,116,794 d \mu^{5}-2,245,432 d^{2} d_{1} \mu^{5} \\
& -780,864 d d_{1} \mu^{5}+1,464,568 d_{1} \mu^{5}+29,285,616 \mu^{5}+23,625 d^{5} \mu^{4} \\
& -1,655,352 d^{4} \mu^{4}+9,527,220 d^{3} \mu^{4}-3,306,270 d^{2} \mu^{4}+2,509,776 d_{1}^{2} \mu^{4} \\
& +122,443,659 d \mu^{4}-2,969,176 d^{2} d_{1} \mu^{4}+29,288,560 d d_{1} \mu^{4} \\
& +17,417,736 d_{1} \mu^{4}+24,043,134 \mu^{4}-61,425 d^{5} \mu^{3}+50,616 d^{4} \mu^{3} \\
& +12,626,676 d^{3} \mu^{3}+3,447,360 d_{1}^{3} \mu^{3}-71,324,322 d^{2} \mu^{3}+37,275,552 d_{1}^{2} \mu^{3} \\
& +188,967,453 d \mu^{3}-31,864 d^{2} d_{1} \mu^{3}+28,661,808 d d_{1} \mu^{3}-61,447,064 d_{1} \mu^{3} \\
& -287,978,454 \mu^{3}-51,975 d^{5} \mu^{2}+1,532,214 d^{4} \mu^{2}+2,083,968 d_{1}^{4} \mu^{2} \\
& -9,689,418 d^{3} \mu^{2}+34,255,872 d_{1}^{3} \mu^{2}+8,294,568 d^{2} \mu^{2}+241,880,112 d_{1}^{2} \mu^{2} \\
& -23,792,511 d^{2}+4,725 d^{5} d_{1} \mu^{2}-61,488 d^{4} d_{1} \mu^{2}-405,720 d^{3} d_{1} \mu^{2} \\
& +6,347,642 d^{2} d_{1} \mu^{2}-34,036,205 d d_{1} \mu^{2}-53,904,858 d_{1} \mu^{2}-333,174,702 \mu^{2} \\
& -4,725 d^{5} \mu+575,712 d^{4} \mu-9,831,060 d^{3} \mu+46,013,184 d_{1}^{3} \mu \\
& +67,722,258 d^{2} \mu+344,248,704 d_{1}^{2} \mu-307,776,015 d \mu+9,450 d^{5} d_{1} \mu \\
& -198,576 d^{4} d_{1} \mu+792,288 d^{3} d_{1} \mu+3,448,340 d^{2} d_{1} \mu-49,503,930 d d_{1} \mu \\
& +140,875,756 d_{1} \mu+560,100,246 \mu+3,150 d^{5}-46,962 d^{4}-8,335,872 d_{1}^{4} \\
& -195,834 d^{3}-17,418,240 d_{1}^{3}+5,150,850 d^{2}-20,975,040 d_{1}^{2}-15,241,836 d \\
& +4,725 d^{5} d_{1}-137,088 d^{4} d_{1}+1,424,808 d^{3} d_{1}-7,789,726 d^{2} d_{1} \\
& +28,911,043 d d_{1}-45,139,458 d_{1}+7,693,848 .
\end{aligned}
$$


Both constants $T_{4}$ and $T_{6}$ are modulo the previous constants and modulo a positive contant.

The period constants $T_{2}, T_{4}$ and $T_{6}$ are polynomials in the variables $d, d_{1}$ and $\mu$. We want to study the zeros $\left(d, d_{1}, \mu\right)$ of $T_{2}, T_{4}$ and $T_{6}$ with $d \geq 5$ an odd positive integer. For doing that we consider the resultant of $T_{2}$ and $T_{4}$ with respect to $\mu$. This resultant is a polynomial $f_{1}$ in the variables $d$ and $d_{1}$. After we consider the resultant of $T_{2}$ and $T_{6}$ with respect to $\mu$. This resultant is a polynomial $f_{2}$ in the variables $d$ and $d_{1}$. The polynomials $f_{1}$ and $f_{2}$ have in common the factors $(1+d)^{2}(5+d) d_{1}^{3}$. We define the polynomials $g_{1}$ and $g_{2}$ as the polynomials $f_{1}$ and $f_{2}$ divided by $(1+d)^{2}(5+d) d_{1}^{3}$. Then we consider the resultant of $g_{1}$ and $g_{2}$ with respect to $d_{1}$. This resultant is a polynomial $h$ in the variable $d$. It easy to check that the unique positive odd integer root $\geq 5$ of the polynomial $h$ is $d=7$. In short the common zeros $\left(d, d_{1}, \mu\right)$ of $T_{2}, T_{4}$ and $T_{6}$ must have either $d_{1}=0$, or $d=7$.

Assume $d_{1}=0$. Then $T_{2}=-3(\mu-1)(d-3+(1+d) \mu)$ and $T_{2}$ divides $T_{4}$ and $T_{6}$. So $d_{1}=0$ and either $\mu=1$ or $\mu=(3-d) /(d+1)$ vanish $T_{2}, T_{4}$ and $T_{6}$. The case $d_{1}=0$ and $\mu=1$ corresponds to the condition (b.1) of Theorem 1 . The case $d_{1}=0$ and $\mu=(3-d) /(d+1)$ corresponds to the condition (b.2) of Theorem 1 . Hence these two conditions are necessary for having an isochronous center.

Assume $d=7$ and $d_{1} \neq 0$. Then

$$
\begin{aligned}
T_{2}= & -12\left(d_{1}^{2}+2 \mu^{2}-\mu-1\right), \\
T_{4}= & -24\left(-156 \mu^{4}-88 d_{1} \mu^{3}+42 \mu^{3}+3 d_{1}^{2} \mu^{2}-28 d_{1} \mu^{2}+72 \mu^{2}\right. \\
& \left.+8 d_{1} \mu+30 \mu-12 d_{1}^{2}+12\right) .
\end{aligned}
$$

Doing the resultant of $T_{2}$ and $T_{4}$ with respect to $d_{1}$ we obtain the polynomial

$$
(\mu-1) \mu(2 \mu+1)\left(10,433 \mu^{3}-3,117 \mu^{2}-3,168 \mu-260\right) .
$$

Substituting $d=7$ and $\mu$ for every one of the six roots of the previous polynomial in $T_{2}, T_{4}$ and $T_{6}$ we get three polynomials in the variable $d_{1}$. Taking into account that $d_{1} \neq 0$, the unique set of the three polynomials which have a common root is the set corresponding to $d=7$ and $\mu=0$. Moreover the common root is $\left(d, \mu, d_{1}\right)=(7,0,-1)$. This case corresponds to the condition (b.3) of Theorem 1 . Hence this condition is necessary for having an isochronous center.

Subcase 2.2.2: $j=1$. By the change of variables in (13) we can rewrite system (1) as

$$
w^{\prime}=i w+(w \bar{w})^{(d-5) / 2}\left[w^{5}+\mu w \bar{w}^{4}+\tilde{D} \bar{w}^{5}\right] .
$$

Since we are in assumptions (a.3) we have that

$$
\tilde{d}_{1}^{2}-\tilde{d}_{2}^{2}=0, \quad \text { that is } \tilde{d}_{1}=\tilde{ \pm} d_{2} .
$$

Here we study the case $\tilde{d}_{2}=\tilde{d}_{1}$. The case $\tilde{d}_{2}=-\tilde{d}_{1}$ provides the same results and can be analyzed in the same way.

Computing $T_{2}$ with these assumptions we have

$$
T_{2}=3(d-5)+24 \mu-3(d+3) \mu^{2}-4(d+5) \tilde{d}_{1}^{2},
$$

modulo a non-zero constant. From now on we write $d_{1}$ instead of $\tilde{d}_{1}$. 
Computing the period constants as in the subcase 2.2.1 we obtain

$$
\begin{aligned}
T_{3}= & 0, \\
T_{4}= & 25 d^{3} \mu^{4}+325 d^{2} \mu^{4}+859 d \mu^{4}+327 \mu^{4}+30 d^{3} \mu^{3}-634 d^{2} \mu^{3} \\
& -4,038 d \mu^{3}-2,270 \mu^{3}-20 d^{3} \mu^{2}-580 d^{2} \mu^{2}-768 d_{1}^{2} \mu^{2}+7,748 d \mu^{2} \\
& +13,588 \mu^{2}-30 d^{3} \mu+634 d^{2} \mu-314 d \mu-27,170 \mu-5 d^{3}+255 d^{2} \\
& +19,200 d_{1}^{2}-4,255 d+15,525, \\
T_{5}= & -23,350 d^{2} \mu^{5}-99,900 d \mu^{5}-89,550 \mu^{5}+36,545 d^{2} \mu^{4}+486,064 d \mu^{4} \\
& +807,687 \mu^{4}-100 d^{4} \mu^{3}+1,510 d^{3} \mu^{3}+46,186 d^{2} \mu^{3}+62,400 d_{1}^{2} \mu^{3} \\
& -849,518 d \mu^{3}-3,233,710 \mu^{3}-100 d^{4} \mu^{2}+5,030 d^{3} \mu^{2}-111,940 d^{2} \mu^{2} \\
& +763,952 d_{1}^{2} \mu^{2}+221,738 d \mu^{2}+6,902,168 \mu^{2}+100 d^{4} \mu-1,510 d^{3} \mu \\
& -44,596 d^{2} \mu-3,400,000 d_{1}^{2} \mu+1,205,546 d \mu-7,343,620 \mu+100 d^{4} \\
& -5,030 d^{3}+97,155 d^{2}+4,101,200 d_{1}^{2}-963,930 d+2,957,025 .
\end{aligned}
$$

These period constants $T_{k}$ are modulo the previous constants $\left\{T_{l}: l=2, \ldots, k-1\right\}$ and modulo a positive contant.

The period constants $T_{2}, T_{4}$ and $T_{5}$ are polynomials in the variables $d, d_{1}$ and $\mu$. We want to study the zeros $\left(d, d_{1}, \mu\right)$ of $T_{2}, T_{4}$ and $T_{5}$ with $d \geq 5$ an odd positive integer. For doing that we consider the resultant of $T_{2}$ and $T_{4}$ with respect to $\mu$. This resultant is a polynomial $f_{1}$ in the variables $d$ and $d_{1}$. After we consider the resultant of $T_{2}$ and $T_{5}$ with respect to $\mu$. This resultant is a polynomial $f_{2}$ in the variables $d$ and $d_{1}$. The polynomials $f_{1}$ and $f_{2}$ have in common the factors $(3+d)^{2} d_{1}^{4}$. We define the polynomials $g_{1}$ and $g_{2}$ as the polynomials $f_{1}$ and $f_{2}$ divided by $(3+d)^{2} d_{1}^{4}$. Then we consider the resultant of $g_{1}$ and $g_{2}$ with respect to $d_{1}$. This resultant is a polynomial $h$ in the variable $d$. It easy to check that the unique positive odd integer root $\geq 5$ of the polynomial $h$ is $d=13$. In short the common zeros $\left(d, d_{1}, \mu\right)$ of $T_{2}, T_{4}$ and $T_{5}$ must have either $d_{1}=0$, or $d=13$.

Assume $d_{1}=0$. Then $T_{2}=-3(\mu-1)(d-5+(3+d) \mu)$ and $T_{2}$ divides $T_{4}$ and $T_{5}$. So $d_{1}=0$ and either $\mu=1$ or $\mu=(5-d) /(d+3)$ vanish $T_{2}, T_{4}$ and $T_{5}$. The case $d_{1}=0$ and $\mu=1$ corresponds to the condition (b.1) of Theorem 1 . The case $d_{1}=0$ and $\mu=(5-d) /(d+3)$ corresponds to the condition (b.2) of Theorem 1. Hence these two conditions are necessary for having an isochronous center.

Assume $d=13$ and $d_{1} \neq 0$. Then

$$
\begin{aligned}
& T_{2}=-24\left(3 d_{1}^{2}+2 \mu^{2}-\mu-1\right), \\
& T_{4}=-768\left(-158 \mu^{4}+125 \mu^{3}+d_{1}^{2} \mu^{2}+36 \mu^{2}-13 \mu-25 d_{1}^{2}+10\right) .
\end{aligned}
$$

Doing the resultant of $T_{2}$ and $T_{4}$ with respect to $d_{1}$ we obtain the polynomial

$$
339,738,624(\mu-1)^{2}(2 \mu+1)^{2}(7 \mu-1)^{2}(34 \mu-5)^{2} .
$$

Substituting $d=13$ and $\mu$ for every one of the four roots of the previous polynomial in $T_{2}, T_{4}$ and $T_{5}$ we get three polynomials in the variable $d_{1}$. Taking into 
account that $d_{1} \neq 0$, the unique set of the three polynomials which have a common root is the set corresponding to $d=13$ and $\mu=1 / 7$. The common roots are $d_{1}=$ $\pm 3 \sqrt{2} / 7$. But computing $T_{6}$ and evaluating it at $\left(d, \mu, d_{1}\right)=(13,1 / 7, \pm 3 \sqrt{2} / 7)$, it is not zero. Consequently there are no more candidates for isochronous centers. This completes the proof of Theorem 1(b).

\section{Proof of Theorems 2 and 3}

We do the proofs of Theorems 2 and 3 in two subsections.

\subsection{Proof of Theorem 2}

Due to the relation between the Liapunov constants and the coefficients of the Poincaré map near the origin of system (1) (see the introduction and the references quoted there) to prove Theorem 2 it is well known that if we can choose $d \geq 29$ odd with the five focal values satisfying $\left|V_{1}\right|<<\left|V_{3}\right|<<\left|V_{5}\right|<<\left|V_{6}\right|<<\left|V_{7}\right|$ and $V_{3} V_{1}<0, V_{5} V_{3}<0, V_{6} V_{5}<0$ and $V_{7} V_{6}<0$, then the cyclicity is five. Moreover if for $d \in\{5,7, \ldots, 27\}$ we can choose the six focal values satisfying $\left|V_{1}\right|<<\left|V_{3}\right|<<\left|V_{5}\right|<<\left|V_{6}\right|<<\left|V_{7}\right|<<\left|V_{9}\right|$ and $V_{3} V_{1}<0, V_{5} V_{3}<0, V_{6} V_{5}<0$, $V_{7} V_{6}<0$ and $V_{9} V_{7}<0$, then the cyclicity is six. From the expressions of the Liapunov constants given in Propositions 5 and 6 it follows after some computations that the previous inequalities hold and consequently Theorem 2 is proved.

\subsection{Proof of Theorem 3}

As in the previous subsection in order to prove Theorem 3 it is well known that if we can choose $d \geq 41$ odd with the six focal values satisfying $\left|V_{1}\right|<<\left|V_{2}\right|<<$ $\left|V_{3}\right|<<\left|V_{6}\right|<<\left|V_{7}\right|<<\left|V_{8}\right|$ and $V_{2} V_{1}<0, V_{3} V_{2}<0, V_{6} V_{3}<0, V_{7} V_{6}<0$ and $V_{8} V_{7}<0$, then the cyclicity is five. Moreover if for $d \in\{7,9, \ldots, 39\}$ we can choose the seven focal values satisfying $\left|V_{1}\right|<<\left|V_{2}\right|<<\left|V_{3}\right|<<\left|V_{6}\right|<<$ $\left|V_{7}\right|<<\left|V_{8}\right|<<\left|V_{10}\right|$ and $V_{2} V_{1}<0, V_{3} V_{2}<0, V_{6} V_{3}<0, V_{7} V_{6}<0, V_{8} V_{7}<0$ and $V_{10} V_{8}<0$, then the cyclicity is six. From the expressions of the Liapunov constants given in Propositions 7 and 8 it follows easily that the previous inequalities hold and so Theorem 3 is proved.

\section{Acknowledgments}

J. Llibre has been supported by the grants MEC/FEDER MTM-03437 and CIRIT-Spain 2005SGR 00550. C. Valls has been partially supported by FCT through CAMGSD, Lisbon.

\section{References}

[1] Andronov, A.A., Leontovich, E.A., Gordon, I.I., Maier, A.G.: Theory of bifurcations of dynamic systems on a plane. Wiley, Nova (1973) 
[2] Bautin, N.N.: On the number of limit cycles which appear with the variation of coefficients from an equilibrium position of focus or center type. Mat. Sbornik 30, 181-196 (1952), Amer. Math. Soc. Transl. 100, 1-19 (1954)

[3] Chavarriga, J., Giné, J.: Integrability of a linear center perturbed by a fourth degree homogeneous polynomial. Publ. Mat. 40, 21-39 (1996)

[4] Chavarriga, J., Giné, J.: Integrability of a linear center perturbed by a fifth degree homogeneous polynomial. Publ. Mat. 41, 335-356 (1997)

[5] Chavarriga, J., Sabatini, M.: A survey of isochronous centers. Qual. Theory Dyn. Syst. 1, 1-70 (1999)

[6] Cheb-Terrab, E.S., Roche, A.D.: An Abel ODE class generalizing known integrable classes. European J. Appl. Math. 14, 217-229 (2003)

[7] Cherkas, L.A.: Number of limit cycles of an autonomous second-order system. Differ. Equ. 5, 666-668 (1976)

[8] Cima, A., Gasull, A., Mañosa, V., Mañosas, F.: Algebraic properties of the Liapunov and period constants. Rocky Mountain J. Math. 27, 471-501 (1997)

[9] Dulac, H.: Détermination et integration d'une certaine classe d'équations différentielle ayant par point singulier un centre. Bull. Sci. Math. Sér (2) 32, 230-252 (1908)

[10] Gasull, A., Llibre, J.: Limit cycles for a class of Abel equations. SIAM J. Math. Anal. 21, 1235-1244 (1990)

[11] Kamke, E.: Differentialgleichungen "losungsmethoden und losungen". Col. Mathematik und ihre anwendungen, 18, Akademische Verlagsgesellschaft Becker und Erler Kom-Ges., Leipzig (1943)

[12] Kapteyn, W.: On the midpoints of integral curves of differential equations of the first degree. Nederl. Akad. Wetensch. Verslag. Afd. Natuurk. Konikl. Nederland, pp. 1446-1457 (Dutch, 1911)

[13] Kapteyn, W.: New investigations on the midpoints of integrals of differential equations of the first degree. Nederl. Akad. Wetensch. Verslag Afd. Natuurk. 20, 13541365 (1912); 21, 27-33 (Dutch)

[14] Malkin, K.E.: Criteria for the center for a certain differential equation. (Russian) Volz Mat. Sb. Vyp. 2, 87-91 (1964)

[15] Pearson, J.M., Lloyd, N.G., Christopher, C.J.: Algorithmic derivation of centre conditions. SIAM Rev. 38, 619-636 (1996)

[16] Poincaré, H.: Mémoire sur les courbes définies par les équations différentielles. Oeuvreus de Henri Poincaré, Vol. I, Gauthiers-Villars, Paris, pp. 95-114 (1951)

[17] Schlomiuk, D., Guckenheimer, J., Rand, R.: Integrability of plane quadratic vector fields. Expo. Math. 8, 3-25 (1990) 
[18] Vulpe, N.I., Sibirskii, K.S.: Centro-affine invariant conditions for the existence of a center of a differential system with cubic nonlinearities. (Russian) Dokl. Akad. Nauk SSSR 301 (1988), 1297-1301; translation in Soviet Math. Dokl. 38, 198-201 (1989)

[19] Zoladek, H.: Quadratic systems with center and their perturbations. J. Differ. Equ. 109, 223-273 (1994)

[20] Zoladek, H.: On a certain generalization of Bautin's theorem. Nonlinearity 7, 273279 (1994)

J. Llibre

Departament de Matemàtiques,

Universitat Autònoma de Barcelona,

Bellaterra, 08193 Barcelona, Catalonia, Spain

e-mail: jllibre@mat.uab.cat

C. Valls

Departamento de Matemática, Instituto Superior Técnico,

Av. Rovisco Pais, 1049-001 Lisbon, Portugal

e-mail: cvalls@math.ist.utl.pt

Received: 16 November 2008.

Revised: 16 April 2009.

Accepted: 17 May 2009. 